www.jmscr.igmpublication.org

Impact Factor (SJIF): 6.379

Index Copernicus Value: 71.58

ISSN (e)-2347-176x ISSN (p) 2455-0450

crossref DOI: https://dx.doi.org/10.18535/jmscr/v6i6.18

Journal Of Medical Science And Clinical Research

\title{
Assessment of Orientation and Fixation of Acetabular Component in Uncemented total Hip Arthroplasty and its Outcome: A Case Series Study
}

\author{
Authors \\ A.Sudharsan ${ }^{*}$, G.A.Rajmohan ${ }^{2}$ \\ ${ }^{1}$ Department of Orthopaedics, Sivagangai Medical College Hospital, Sivagangai, Tamilnadu, India \\ ${ }^{2}$ Department of Orthopaedics, Thanjavur Medical College Hospital, Thanjavur, Tamilnadu, India
}

\begin{abstract}
Introduction: Fixation of porous im plant depends on bone in growth and bone on growth in hydroxyapatite coated acetabular implant, CT scans were played an important role in evaluating acetabular cup position precisely. Formation of new bone in prosthetic bone interface (osteointegration) is a good sign of stability in uncemented total hip replacement.

Aim: A study to analyse the importance of acetabular component orientation and fixation for the outcome of uncemented total hip arthroplasty.

Meterial \& Methods: 18 to 60 years of aged fifteen patients underwent uncemented total hip arthroplasty through posterior approach during June2013 to March2015.Periodic evaluation of osteointegration and orientation of acetabular component was done clinically and radiologically using CT scan.

Results: Harris Hip Score improved from 29.9 preoperative to 91.4 postoperatively in our study with one case of deterioration but gave fair result. Mean acetabularante version and inclination was 21 degree and 41 degree respectively.

Conclusion: In this short term study clinical outcome correlated well with radiological appearance and had comparable results with previous studies.

Keywords: osteointegration, acetabular component, anteversion, inclination, arthroplasty.
\end{abstract}

\section{Introduction}

A good long-term outcome of total hip replacement depends on accurate acetabular cup position. CT scans were played an important role in evaluating acetabular cup position precisely. Fixation of porous implant depends on bone in growth and bone on growth in biologically active implant surface coated with hydroxyapatite ${ }^{1}$, otherwise osteo- integration will not occur comfortably in uncemented total hip arthroplasty. Pores of size100 to $400 \mu \mathrm{m}$ is essential for bone ingrowth $^{2}$. Immediate stability of the fixation is the important factor which gives good fixation in long term. Micromotion between the implant and bone less than $50 \mu \mathrm{m}$ facilitate bone in growth ${ }^{2}$. Hydroxy apatite coated acetabular implant in uncemented total hip arthroplasty in acetabular sockets get bone in growth and render good clinical results. Moreover post operative bone loss is expected inosteoporotic uncemented total hip arthroplasty. Hence osteo integration (formation of new bone in prosthetic bone interface) is a good sign of stability in uncemented total hip replacement. 


\section{Material \& Methods}

Prospective evaluation of fifteen patients undergone uncemented total hip replacement in the Department of Orthopaedics and Traumatology, Thanjavur medical college hospital, Thanjavur Tamilnadu. 18 to 60 years old patients were subjected to our study and the indications were healthy patients with good bone stock without any septic foci, avascular necrosis head of femur, old fracture neck of femur non union and chronic arthritis of hip, during the period of June 2013 to March 2015 for evaluating acetabular orientations and fixation of acetabular cup. The implant used in our study has sand blasted outer convex surface of titanium cup facilitates osteointegration with openings for $6.5 \mathrm{~mm}$ titanium cancellous screws countersunk in proximal half inserted towards the sacroiliac joint for additional fixation and in distal half of the cup has no screw openings. The sharp edged elevations facilitate penetration into the cancellous bone when impaction and this increases the primary stability and prevent rotation and tilting which favours subsequent osteointegration, implanted cup shell with somewhat greater diameter by $2 \mathrm{~mm}$ than the previously reamed acetabulam also provides primary stability. The standard cup was available in $2 \mathrm{~mm}$ steps with diameter from $44 \mathrm{~mm}$ to $62 \mathrm{~mm}$. The high density polyethylene insert has a projection on its convex surface which fit into the central hole and can be used as a guidefore insertion when impacted the shell. Patients were admitted at Thanjavur Medical College Hospital Orthopaedic Department and underwent all necessary investigations. They were examined for limb length discrepancy, fixed deformities, active and passive range of movements at the affected hip, distal neurovascular deficit, status of other joints and any focus of infection (skin, dental and urinary tract) and were subjected to preoperative Harris Hip Scoring system analysis ${ }^{3}$. They were obtained consent and assessment for suitable anaesthesia for total hip replacement. All the patients were operated through posterior approach.
Postoperatively they were transported in supine with operated limb abducted by a pillow and were connected to multipara monitor to monitor vitals, urine output, soakage and drain output were monitored. They were encouraged ankle and toe mobilization in bed as soon as the motor function recovered. Analgesics, antacids and parental antibiotics were continued for 48 to $72 \mathrm{hrs}$ later they were put on oral antibiotics for 5 to 7 days, thrombo prophylaxis of subcutaneous low molecular heparin was started $12 \mathrm{hrs}$ after the surgery. Hemoglobin and packed cell volume (PCV) were checked and blood transfusion was decided if $\mathrm{Hb}$ less than $9 \mathrm{mg} \%$ and PCV less than $27 \%$. Suction drain was removed after $24 \mathrm{hrs}$ as the drain was less than $100 \mathrm{ml}$. Physiotherapy was started in bed on the first postoperative day with strictly advised to continue the abduction pillow and not to lie on operated side for 3 to 6 weeks. They were made to stand up and walk with partial weight bearing using walker on third postoperative day. A high western commode seat toilet was advised and were discharged after removal of sutures. Advised care at home was continue supervised physiotherapy, continue walker for $6-12$ weeks and followed by cane in the contralateral side, avoid stair climbing upto612 weeks. Harris Hip Scoring system ${ }^{3}$ was used for clinical outcome analysis. These patients had follow up with radiographs at regular intervals (immediate postoperatively, 1 month, 3 months, 6 months, 12 months and 18 months). Collected data was observed statistically.

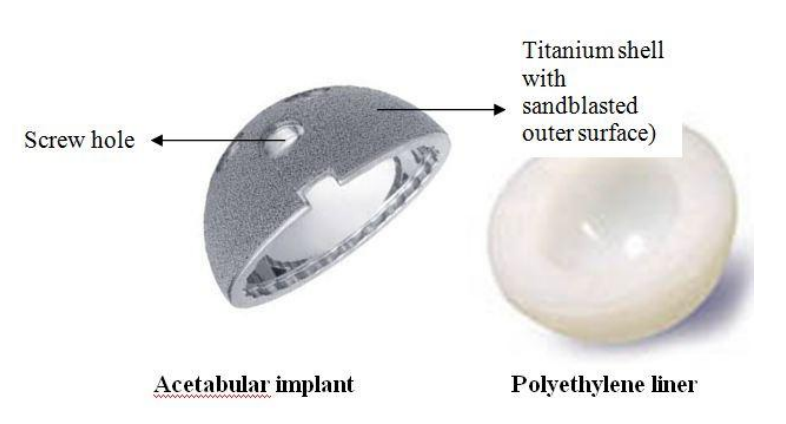

\section{Operative procedures:}

Patients were placed in lateral position with the affected limb on the upper aspect, stabilized in place by supports, clamps and sandbags. $10 \mathrm{~cm}$ of 
straight skin incision started $6 \mathrm{~cm}$ proximal to tip of greater trochanter and extended towards the greater trochanter and then along the lateral border of thigh with the affected hip flexed to 45 degree. The facialata was divided over the greater trochanter and carried in both directions in line with skin incision. The fibres of gluteus maximus were separated by blunt dissection to expose the short external rotators. The sciatic nerve came in path on the lateral side, while the inferior gluteal vessels were near the proximal most portion of the incision were taken with great care during dissection. The short rotators were divided close to their insertion $1 \mathrm{~cm}$ from the trochanter which exposed the hip joint capsule and was incised in a $\mathrm{T}$ fashion. The psoas tendon was divided off the lesser trochanter if necessary Internal rotation of the leg using the bent knee as a lever arm, hip was dislocated, which gives an adequate exposure of acetabulam by using Hohmann retractors. The short lateral rotators were reattached us in the stay sutures applied earlier.

\section{Results}

Clinical Evaluation: Among the 15 patients the overall improvement in Harris hip score was from 29.9 preoperatively to 91.4 post operatively and only in one case we observed deterioration in Harris hip score, but it still gave fair result. The prevalence of anterior thigh pain was $6.7 \%$ in one year follow up evaluation. Patients were evaluated for complications like intra operative periprosthetic femoral fractures, superficial infection, dislocations, limb length discrepancy wasting of thigh muscles and found no cases with dislocation and periprosthetic fractures. The clinical result of HHS was evaluated for statistical significant using Wilcoxan Signed Rank Test in three stages from immediate, 3 months and 12 months followup period.

Radiographic Evaluation: Radiographs were ordered in each hip immediately after the operation, 1 month, 3 months, 6 months, 12 months and 18 months intervals. These are the standard antero posterior radiograph of pelvis with both hips, measurements of cup inclination on AP radiographs were reliable and accurate compared with CT measurements. The radiographs were cautiously reviewed to determine the presence and progression of radiodense and radiolucent lines, the position and migration of the acetabularcup. Three zones (DeLee and Charnley ${ }^{4}$ ) along the acetabular component was assessed for the presence and progressions of radio dense and radiolucent lines along the acetabular component and there was no significant radiolucency in zone 1 and zone 2, butin $6.7 \%$ (case-3) of our patients there was a significant radiolucency in zone 3 , the non- weight bearing area of the implant. There was no significant fresh bone formation in acetabular and bone interface.

CT Scan Evaluation CT Scans were done in all the patients immediate postoperatively and also with follow up dates. These patient had regular AP radiographs and transverse view of CT scan, there were scattered radiation lines produced by metal implants and were suppressed in the newer CT scanners. This allowed the images of the implanted cup and hence their measurements more accurate according to previous studies but due to metal artifact bone in growth was difficult to interprete, while doing cross sections in transverse planes over the zones described by DeLeeandCharnley ${ }^{4}$ in CT scan. In immediate post operative CT Scan primary press fit of components were noted by impaction of the triangular projections into the acetabulum. In post operative follow up CT Scans the presence of any new bone in prosthetic bone interface or osteolysis over the acetabular component were analysed. The available CT scans did not show any new bone formation around acetabular component in our short term of one year study also no comparative analysis was available in the literatures to retrieve the results which are the limitation in our study. Radiolucent areas were seen in one patient $(6.7 \%)$ and the remaining $94 \%$ of patients showed no significant osteolysis, but 
had a good clinical outcome with average Harris Hip Score of 92 also the $6.7 \%$ of patients had significant osteolysis with fair clinical outcome with average Harris Hip Score of 76.

\section{Case- 1}

Preoperative Clinical Picture
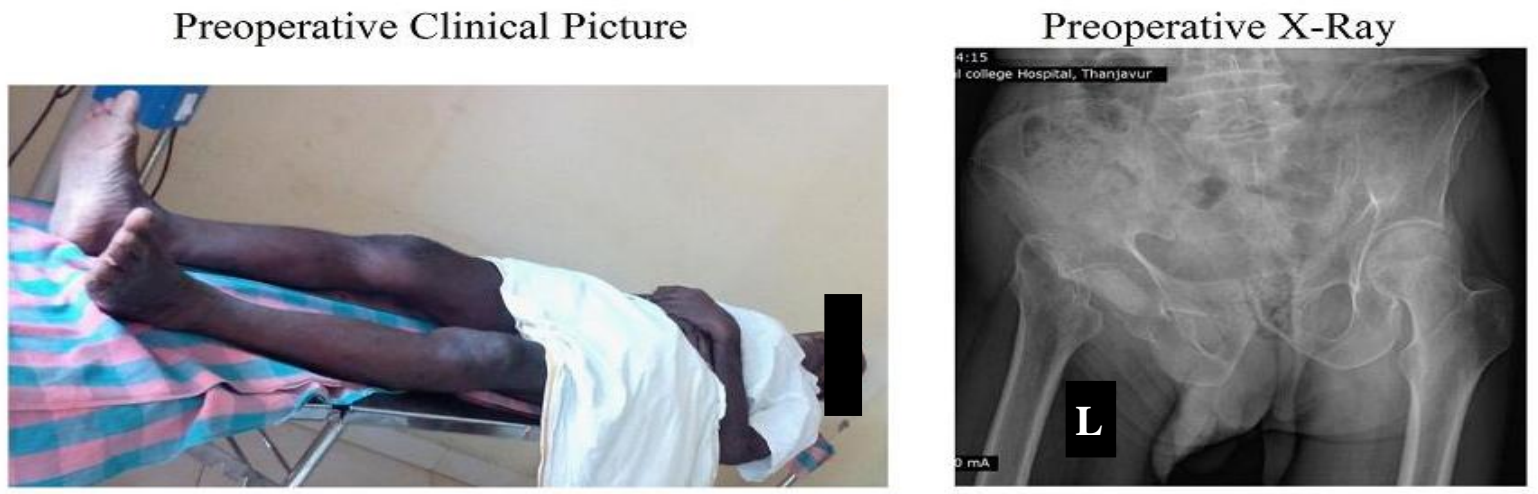

Postoperative Clinical Assessment
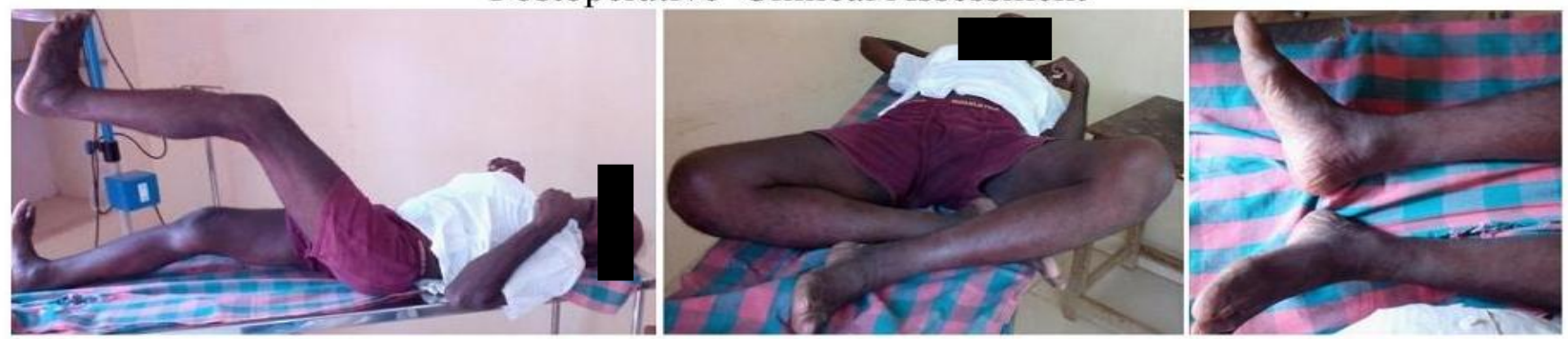

Postoperative Radiological Assessment
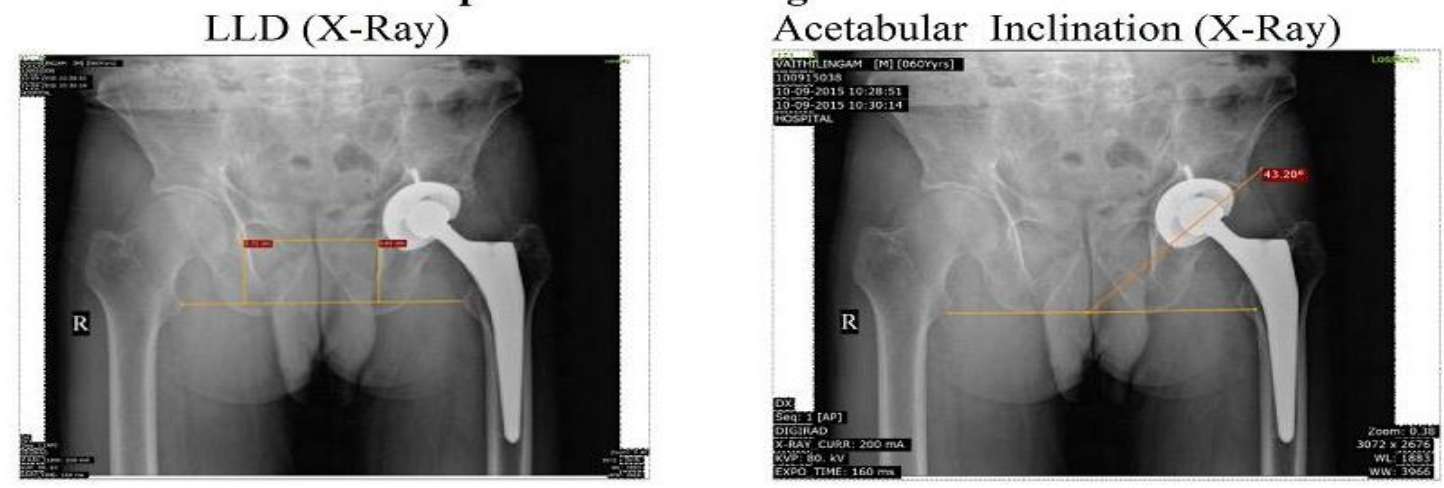

Acetabular Cup Anteversion (Ct)

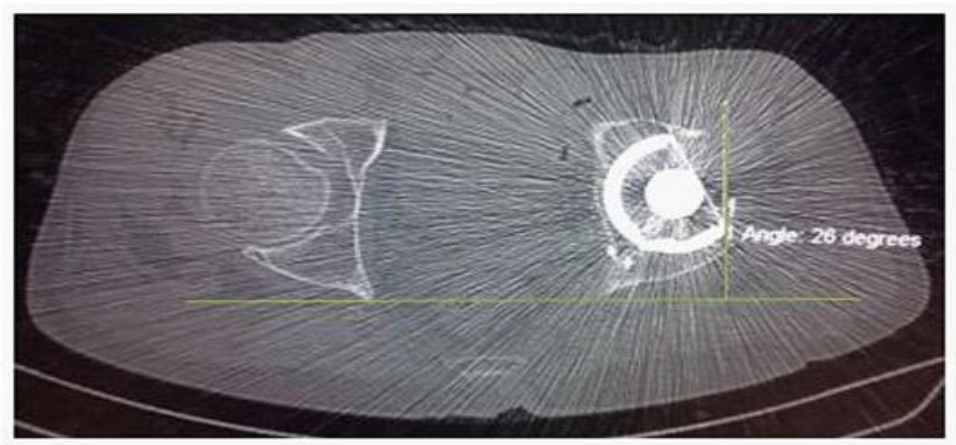




\section{Case-2}

Preoperative X-ray Postoperative Clinical Assessment

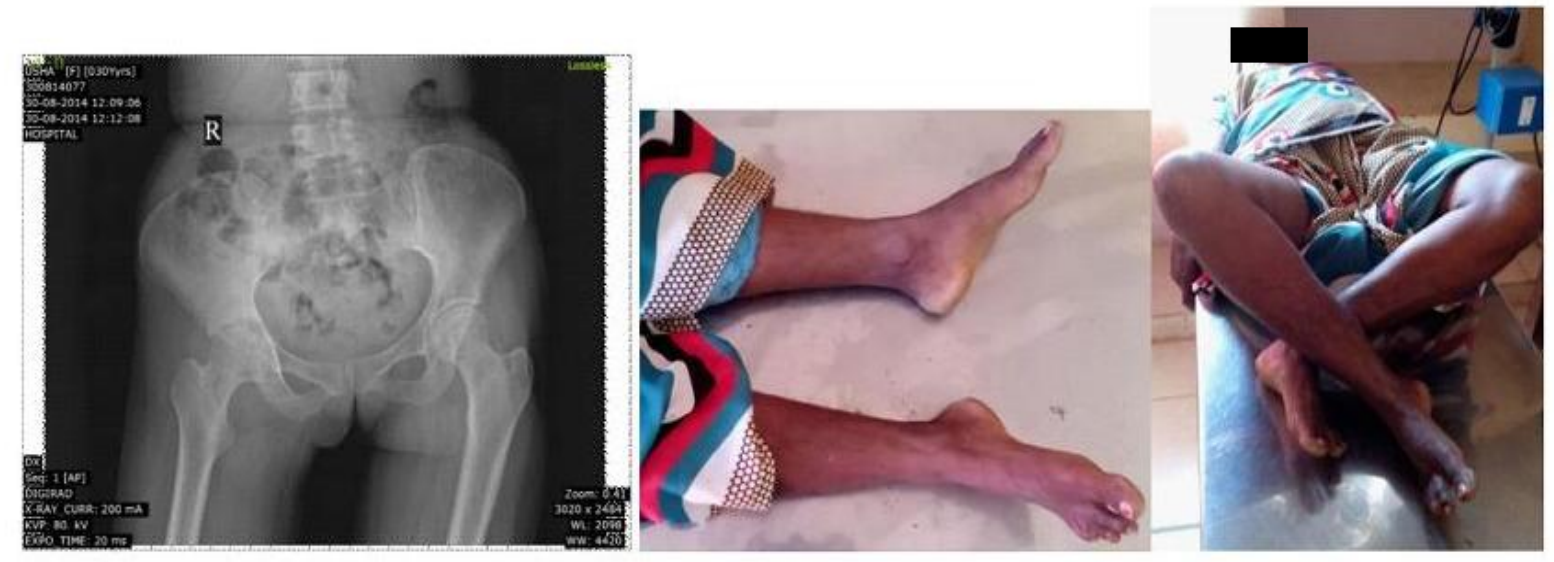

Postoperative Radiological Assessment

LLD (X-Ray)

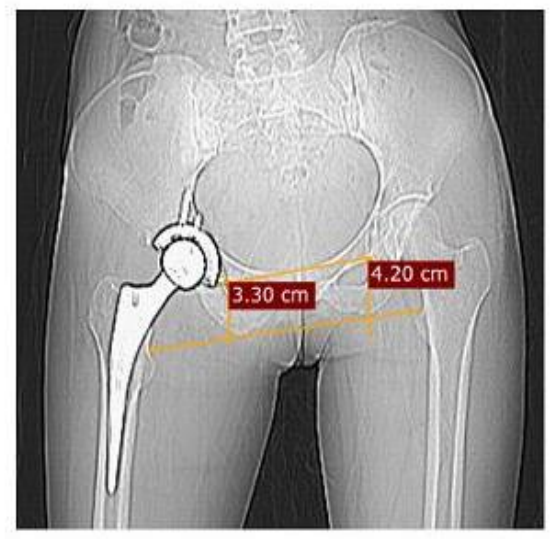

Acetabular Inclination (X-Ray)

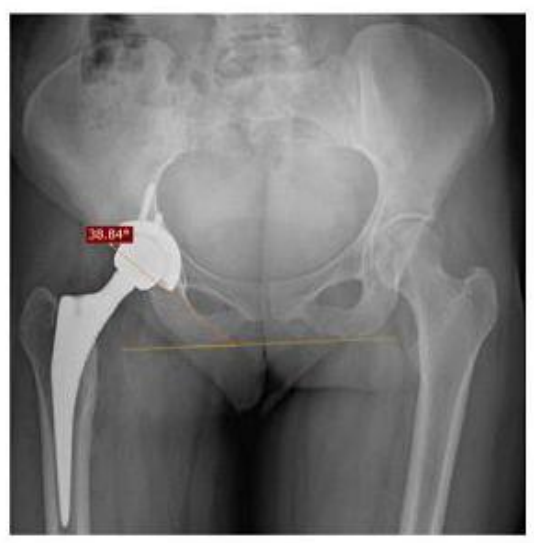

Acetabular Cup Anteversion (Ct)

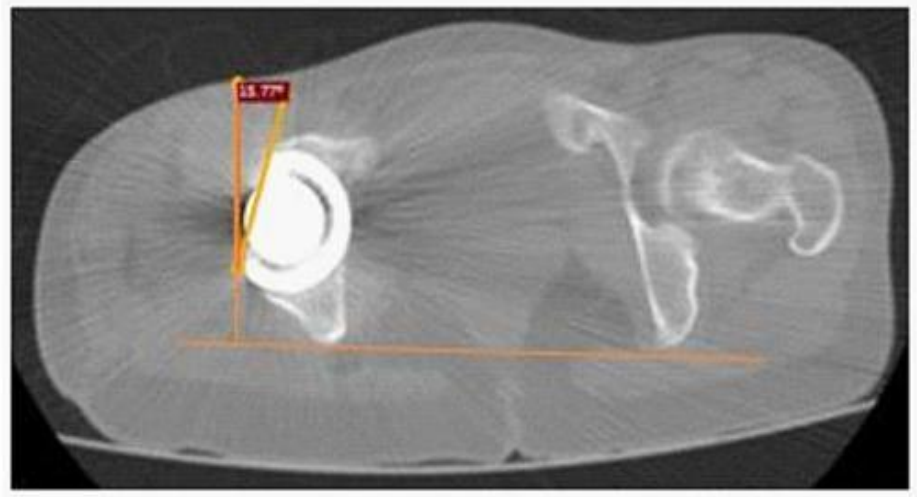




\section{Case 3}
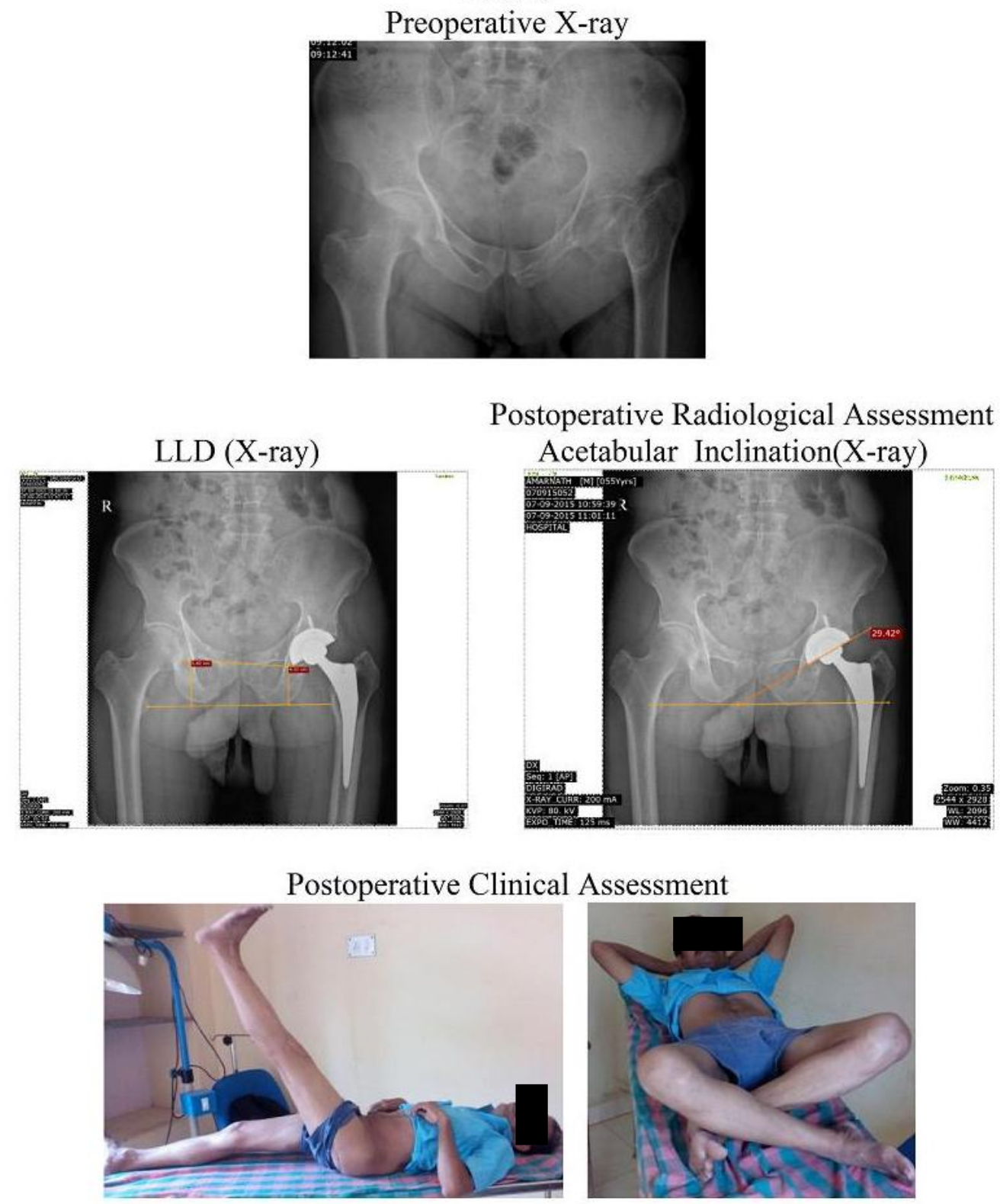

Acetabular Cup Anteversion with Radiolucent line (Arrow)

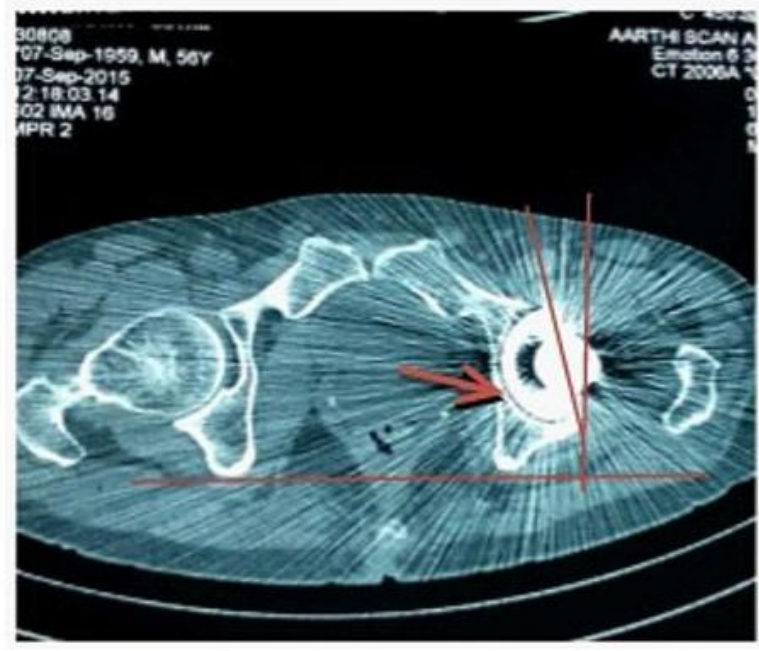


Table: 1 Clinical variables

\begin{tabular}{|l|c|c|}
\hline Variables & Mean +/- SD & Median(Range) \\
\hline Age(Years) & $42.47+/-15.43$ & $45(18-60)$ \\
\hline Gender $(\mathrm{M}: \mathrm{F})$ & $10: 05$ & \\
\hline LLD $(\mathrm{Cm})$ & $0.35+/-0.29$ & $0.2(0.1-1.09)$ \\
\hline
\end{tabular}

Table: 2 Mean measuring values

\begin{tabular}{|l|c|c|}
\hline Parameter & Mean+/- SD & Median(Range) \\
\hline Cup Size (mm) & $50.8+/-3.84$ & $50(46-58)$ \\
\hline Anteversion (Deg) & $21.57+/-6.68$ & $21(11.2-32)$ \\
\hline Inclination (Deg) & $41.83+/-10.07$ & $40.6(26.7-64.7)$ \\
\hline
\end{tabular}

Table: 3 Indications

\begin{tabular}{|l|c|c|}
\hline Indications & No of Cases & Percentage \\
\hline AVN Femoral Head & 2 & 13.3 \\
\hline Fracture Neck of Femur Nonunion & 10 & 66.6 \\
\hline Chronic Arthritis & 3 & 20 \\
\hline
\end{tabular}

Table: 2 Wilcoxon Signed Rank Test

\begin{tabular}{|l|l|l|l|}
\hline \multirow{2}{*}{ Variable } & \multicolumn{2}{|l|}{ Mean \pm SD (Minimum- Maximum) } & \multirow{2}{*}{ P value } \\
\cline { 2 - 3 } & PRE OP & HHS IMMED POSTOP & \\
\hline HH Score & $29.93 \pm 9.42(18-41)$ & $36.33 \pm 0.98(35-37)$ & 0.053 \\
\hline
\end{tabular}

\begin{tabular}{|l|c|c|c|}
\hline \multirow{2}{*}{ Variable } & \multicolumn{2}{|c|}{ Mean \pm SD (Minimum- Maximum) } & \multirow{2}{*}{ P value } \\
\cline { 2 - 3 } & PRE OP & HHS 3M & \\
\hline HH Score & $29.93 \pm 9.42(18-41)$ & $73.67 \pm 2.19(70-76)$ & 0.001 \\
\hline
\end{tabular}

\begin{tabular}{|l|c|c|c|}
\hline \multirow{2}{*}{ Variable } & \multicolumn{2}{|c|}{ Mean \pm SD (Minimum- Maximum) } & \multirow{2}{*}{ P value } \\
\cline { 2 - 3 } & PRE OP & HHS 1Year & \multirow{2}{*}{0.005} \\
\hline HH Score & $\begin{array}{c}29.93 \pm 9.42 \\
(18-41)\end{array}$ & $\begin{array}{c}89.8 \pm 5.29 \\
(76-96)\end{array}$ & \\
\hline
\end{tabular}

Interpretation for Above 3 tables

The pre-operative mean $\mathrm{HH}$ score was $29.93 \pm$ 9.42 with a range of 18 to 41 increased to $36.33 \pm$ 0.98 at immediate post-operative.. This increase shows trend towards statistical significance $(\mathrm{P}=0.053)$.

Three months follow-up mean $\mathrm{HH}$ score was $73.67 \pm 2.19$ with a range of 70 to 76 . The improvement observed at 1 month from preoperative HHS show a statistical significance $(\mathrm{P}=0.001)$.

At the end of one year the mean $\mathrm{HH}$ score improved to $89.8 \pm 5.29$ compared to $29.93 \pm 9.42$ at pre-operative. This increase from pre-operative HHS show a statistical significance improvement $(\mathrm{P}=0.005)$.

\section{Discussion}

Pidhorz et $a l^{5}$ proved radiological and histological relationship between bone and implant in retrieved acetabular cup prosthesis and noticed bone ingrowth. This new bone formation initiatesas early as one month postoperatively which progressively increases and results coverage of porous coating surfaces with bone also complete incorporation of the implant occurred at an average of 41 months ${ }^{1}$.He also mentioned that a membrane like structure was formed around the prosthesis because of migration of particulate wear common in uncemented component than cemented where particulate debris are usually sealed off. Engh and Bobin ${ }^{6}$ classified implant fixation based on roentegenographic examination $\mathrm{as}^{4}$ (1) bone ingrowth(2) stable fibrous and (3) unstable. one $\mathrm{mm}$ radio lucent line formed over 
the cup without any migration of the implant is fibrous in growth and was usually stable. Incomplete sclerosis and osteolytic lines close to the impant designate instability, the radiolucent lines in the interface between implant and bone with migration of implant indicate loosening. Since plain radiography misjudge the degree of osteolysis and osteointegration, CT scan assists in differentiating the type of lesions, three stages such as Initial inflammatory phase, reparative woven bone (one to two weeks) and lamellar remodeling bone (four weeks) occur at the interface of implant porous surface and bone to retain the stability. Improvement in bone in growth otherwise called osteointegration depends on the accomplishing stability between the bone and the implant. Experimental studies show that it has been found that calcium phosphate treated porous surface gives systemically enhanced good fixation and apposition of prosthesis to bone. Also it is obvious that factors affecting fracture healing can also inhibit bone ingrowth in uncemeted total hip replacement components for example drugs like indomethacin, bisphophanates, low dose radiation therapy which are used to treat heterotrophic ossification and increased motion in the interface between implant and bone can reduce the bone ingrowth even upto 75 percent.

In total hip replacement preoperative templating is essential to assess lateral and anterior inclination of the cup and is important to avoid postoperative problems like impingement, dislocation, wearing and loosening. The angle formed between the long axis of the cup ellipse and theinter-teardrop line on AP radiographs is cup inclination and the angle between a line joining the lateral anterior and posterior margins of the acetabular component and the perpendicular line connecting the posterior margins of both acetacula is cup anteversion ${ }^{7}$. By correcting pelvic rotation, abduction/adduction and tilt by placing the patient on CT table and squaring the pelvis anterior inclination or anteversion (CT scan) and lateral (roentgenogram) inclination were measured. However the observations and interpretations of the radiographic measurements depends on reviewer which is a limitation of our study. According to Charnley $^{8} 0$ degree of anterior inclination was sufficient, Coventry ${ }^{9}$ proposed 40 degree of lateral and 15 degree of anterior inclination, where as Harris $^{10}$ proposed 30 degree of lateral and 20 degree of anterior inclination. Based on these studies, cup inclination angle ranges from 30 to 50 degree lateral and 0 to 30 degree anterior is suggested. In our study we had mean acetabularante version of 21.5 degree and lateral inclination of 42 degree comparable to previous studies. In patients with unilateral hip disorder healthy side can be taken as reference point. However, in patients with bilateral hip joint disorder there is no reference point apart from the height of the teardrop.

Ranawat et al $^{11}$ advocate true acetabular region for cup position and the approximate femoral head center as the reference using tear drop (intersection of Senton's line and Kohler's line). The cup size was chosen to fit the anteroposterior acetabular diameter and in contact with medial wall of the acetabular floor and the inferior edge at the level of the inferior acetabular margin.

\section{Conclusion}

Though long term follow up is essential to assess the potential mechanism of acetabular component failure, unusual polyethylene wear, failure of locking mechanism, detachment of the liner and pelvic osteolysis our study shows no such problems. CT Scan is better than radiograph in assessing the new bone formation around prosthesis. Our short term study of one particular type of uncemented total hip replacement system with hydroxyapatite coated sandblasted surface titanium acetabular cup implanted in pressfit technique has comparable values of acetabular orientation with previous studies and correlated well clinically and radiologically. Howerver there were no evidence of osteointegration in any case but radioluceny over the acetabular cup in single case, which also gave good clinical result. Uncemented total hip arthroplasty gave acceptable 


\section{JMSCR Vol||06||Issue||06||Page 100-108||June}

results in otherwise disabling condition of hip. Also at one year follow up we found no difference in clinical and radiological outcome. CT scan is better than roentgenogram in assessing the new bone formation and orientation.

\section{Reference}

1. BN Weissman, Imaging of total hip replacement, Radiology 1997;202:611

2. Paul RT Kuzyk and Emil H Schemitsch The basic science of periimplant bone healing Indian J Orthop. 2011 Mar-Apr; 45(2): 108-115.

3. Anna nilsdotter and Ann bremander, Measures of Hip function and symptoms, Arthritis care \& Research vol63,no.S11, nov201,ppS200-207

4. DeLee J.G, and Charnley, John, Radiolical demarcation of cemented sockets in total hip replacement Clin Orthop.,121;2032,1976.

5. Pidhorz LE, UrbanRM, JacobsJJ, Sumner DR, Galante JO. Aquantitative study of bone and soft tissues in cementless porouscoatedacetabular components retrieved at autopsy. J. Arthroplasty, 8(2):213-225, 1993

6. Engh CA, Bobyn JD, Glassman AH. Porous coated hip replacement. The factors governing bone ingrowth, stress shielding, andclinical results. J Bone Joint Surg Br.1987;69:45-55.

7. Wines AP, McNicol D. Computed tomography measurement of the accuracy of component version in total hip arthroplasty. J Arthroplasty. 2006;21(5) :696-701. doi: 10.1016/j.arth.2005.11.008.

8. Charnley J. Total hip replacement by low friction arthroplasty. Clin Orthop Relat Res.1970;(72):7-21.

9. Coventry MB. Late dislocations in patients with Charnley total hip arthroplasty. J Bone Joint Surg Am.1985;67(6):832-841.
10. Harris WH. Advances in surgical technique for total hip replacement without and with osteotomy of the greater trochanter. Clin Orthop Relat Res. 1980;146(2):188-204.

11. Ranawat CS, Dorr LD, Inglis AE. Total hip arthroplasty in protrusion acetabula of rheumatoid arthritis. J Bone Joint Surg Am.1980,62(7): 1059-1065. 